Published in final edited form as:

Chem Mater. 2019 October 8; 31(19): 8035-8043. doi:10.1021/acs.chemmater.9b02485.

\title{
Rapid Diels-Alder Cross-linking of Cell Encapsulating Hydrogels
}

\author{
Christopher M. Madl ${ }^{*} \dagger, \ddagger$ Sarah C. Heilshorn§ \\ tDepartment of Bioengineering, Stanford University, Stanford, California 94305, United States \\ ‡Baxter Laboratory for Stem Cell Biology, Department of Microbiology \& Immunology, Stanford \\ University, 269 Campus Drive CCSR 4215, Stanford, California 94305, United States \\ §Department of Materials Science \& Engineering, Stanford University, Stanford, California 94305, \\ United States
}

\begin{abstract}
Recent efforts in the design of hydrogel biomaterials have focused on better mimicking the native cellular microenvironment to direct cell fate. To simultaneously control multiple material parameters, several orthogonal chemistries may be needed. However, present strategies to prepare cell-encapsulating hydrogels make use of relatively few chemical reactions. To expand this chemical toolkit, we report the preparation of hydrogels based on a Diels-Alder reaction between fulvenes and maleimides with markedly improved gelation kinetics and hydrolytic stability. Fulvene-maleimide gels cross-link up to 10-times faster than other commonly used DA reaction pairs and remain stable for months under physiological conditions. Furthermore, fulvenemaleimide gels presenting relevant biochemical cues, such as cell-adhesive ligands and proteolytic degradability, support the culture of human mesenchymal stromal cells. Finally, this rapid DA reaction was combined with an orthogonal click reaction to demonstrate how the use of selective chemistries can provide new avenues to incorporate multiple functionalities in hydrogel materials.
\end{abstract}

\section{Graphical Abstract}

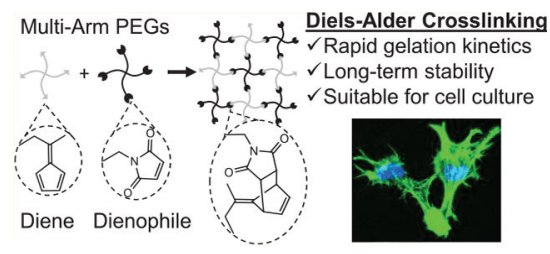

\footnotetext{
*Corresponding Author: cmadl@stanford.edu.

Supporting Information

The Supporting Information is available free of charge on the ACS Publications website at DOI: 10.1021/acs.chemmater.9b02485. Synthetic protocols and characterization for diene- and dienophile-functionalized materials, representative gelation time sweep of 4 arm PEG-methylfuran hydrogel, representative frequency sweeps reporting storage $\left(\mathrm{G}^{\prime}\right)$ and loss $\left(\mathrm{G}^{\prime \prime}\right)$ moduli for 4-arm PEG gels, ${ }^{1}$ H NMR analysis of hydrogel cross-linking and degradation for furan- and methylfuran-based gels, ${ }^{1} \mathrm{H}$ NMR analysis of hydrogel cross-linking and degradation for fulvene-based gels, and summary of plateau storage moduli ( $1 \mathrm{~Hz}, 10 \%$ strain) for PEG-based Diels-Alder hydrogels (PDF)

The authors declare no competing financial interest.
} 


\section{INTRODUCTION}

Hydrogel biomaterials are commonly used as mimics of the native extracellular matrix (ECM) due to their high water content and biocompatibility. ${ }^{1,2}$ Numerous studies in recent years have revealed the ability of several hydrogel properties to regulate cellular behaviors, including matrix stiffness, viscoelasticity, cell-adhesive ligand concentration, growth factor presentation, and matrix degradation. ${ }^{3}$ To facilitate cross-linking and chemical functionalization of hydrogels for cell encapsulation, reactions from the broad class of "click chemistry" reactions have grown in popularity due to their robust and selective reactivity in the presence of water and living cells. ${ }^{4-6}$ These reactions are characterized by rapid reaction kinetics and good hydrolytic stability, both of which are essential qualities for cross-linking reactions used to generate cell-encapsulating hydrogels.

The classic Diels-Alder (DA) reaction represents an underutilized cross-linking chemistry in regards to cell-encapsulating hydrogels. While DA hydrogels have shown promise as drug delivery platforms, ${ }^{7-14}$ their use as three-dimensional (3D) tissue engineering scaffolds has been limited to a small subset of polymeric hydrogel materials and cell culture applications. 15,16 This is likely due in part to the relatively slow gelation kinetics of the commonly-used DA reaction pair that employs furan as the diene and maleimide as the dienophile. Despite the substantial acceleration of the DA reaction in water, ${ }^{17}$ furan-maleimide gels can take hours to cross-link under physiological conditions that are suitable for cell encapsulation. $7,11,16,18$ Such long gelation times often result in an inhomogeneous cell distribution within the hydrogels due to sedimentation and decreased cell viability if the cross-linking reaction is carried out in a simple saline buffer as opposed to cell culture medium. ${ }^{16}$ Furthermore, the reversibility of the DA reaction pair can result in swelling and substantial degradation of furan-maleimide gels over time. ${ }^{19}$ Under physiological conditions, the cross-links can undergo a retro-DA reaction to yield the maleimide starting material, which is prone to hydrolysis. Thus, the cross-links are gradually broken, resulting in changing material properties such as hydrogel stiffness and mesh size that can become confounding variables in cell culture studies.

Previous mechanistic studies have revealed correlations between the electronic structure of substituted furan dienes and the rates of both DA and retro-DA reactions with maleimides. ${ }^{20-23}$ While previous studies focused on altering the substituents of the furans, for instance, by adding an electron-donating methyl group, we reasoned that replacing the furan diene with an even more electron-rich cyclic diene, such as a fulvene, would further enhance the reaction rate of the DA reaction (Figure 1). Fulvenes are commonly used as dienes in organic synthesis and have been previously used to prepare self-healing, polymeric materials. ${ }^{24,25}$ In this work, fulvenes are paired with maleimide dienophiles to rapidly crosslink multi-arm poly(ethylene glycol) (PEG) hydrogels and hybrid PEG-engineered protein hydrogels with markedly improved stability for cell encapsulation (Scheme 1). 


\section{EXPERIMENTAL SECTION}

\section{General Considerations.}

All reagents were purchased from Sigma-Aldrich or Fisher Scientific and used without further purification, unless otherwise noted. Multi-arm PEG starting materials were purchased from JenKem Technology. NMR spectroscopy was performed on a Varian Inova $500 \mathrm{MHz}$ spectrometer in the Stanford University NMR Facility. Chemical shifts were referenced to the residual solvent peak. For NMR of modified elastin-like proteins (ELPs), a drop of $\mathrm{D}_{2} \mathrm{O}$ was added to the solution of ELP in DMSO- $d_{6}$ to suppress the signals from the amide protons.

\section{Synthesis of 4-(Cyclopenta-2,4-dien-1-ylidene)pentanoic Acid.}

This synthetic protocol was adapted from previously published procedures. ${ }^{24,26}$ Cyclopentadiene was freshly distilled from dicyclopentadiene. Briefly, dicyclopentadiene was heated to $170{ }^{\circ} \mathrm{C}$ with stirring in a round-bottom flask equipped with a Vigreaux distilling column that was attached via a glass joint to a Liebig condenser. The condenser was further equipped with a second, round-bottom flask submerged in a dry ice/isopropanol bath to prevent re-dimerization of the cracked cyclopentadiene. The collected cyclopentadiene was used immediately. Levulinic acid $(4.0 \mathrm{~g}, 35 \mathrm{mmol})$ and cyclopentadiene (7.3 mL, $5.9 \mathrm{~g}, 87.5 \mathrm{mmol}$ ) were dissolved in $35 \mathrm{~mL}$ of methanol. This mixture was degassed with nitrogen for $10 \mathrm{~min}$. A catalytic amount of pyrrolidine $(20 \mathrm{~mol} \%, 0.5 \mathrm{~g}, 7$ $\mathrm{mmol}$ ) was dissolved in triethylamine $(5.1 \mathrm{~g}, 50 \mathrm{mmol})$ and added dropwise to the stirring reaction mixture over the course of $10 \mathrm{~min}$. The resulting mixture was degassed for an additional $5 \mathrm{~min}$, and the reaction was allowed to proceed at room temperature under a nitrogen atmosphere. The reaction progress was monitored by thin-layer chromatography, which indicated that the starting material was consumed within $1 \mathrm{~h}$. At that point, the reaction mixture was diluted with diethyl ether $(100 \mathrm{~mL})$ and washed with $0.5 \mathrm{M}$ aqueous hydrochloric acid $(100 \mathrm{~mL})$. The organic layer was then extracted with $0.1 \mathrm{M}$ aqueous sodium hydroxide $(2 \times 100 \mathrm{~mL})$. The combined aqueous layers were acidified to $\mathrm{pH} \approx 1$ with hydrochloric acid and then extracted into dichloromethane $(3 \times 200 \mathrm{~mL})$. The combined organic layers were dried over magnesium sulfate, filtered, and concentrated by rotary evaporation to yield a yellow oil. The crude product was purified by silica chromatography. Silica gel (high-purity grade, pore size $60 \AA$, 230-400 mesh) was wetpacked in a flash chromatography column in ethyl acetate. The crude product was loaded directly onto the column, and the desired product was eluted with ethyl acetate. Fractions containing the product were combined and concentrated in vacuo to afford 4(cyclopenta-2,4-dien-1-ylidene)pentanoic acid as a yellow solid (1.8 g, $11 \mathrm{mmol}, 31.4 \%$ yield). ${ }^{1} \mathrm{H}$ NMR (500 MHz, $\left.\mathrm{CDCl}_{3}\right) \delta$ ppm: 10.78 (br s, $\left.1 \mathrm{H}\right), 6.51$ (m, 4H), 2.88 (t, $J=7.6$ $\mathrm{Hz}, 2 \mathrm{H}), 2.61$ (t, $J=7.6 \mathrm{~Hz}, 2 \mathrm{H}), 2.22$ (s, 3H); ${ }^{13} \mathrm{C} \mathrm{NMR}\left(126 \mathrm{MHz}, \mathrm{CDCl}_{3}\right) \delta \mathrm{ppm}: 178.64$, $149.69,143.40,131.57,131.41,120.75,120.16,33.38,31.48,20.56$.

\section{Synthesis of SMCC.}

This synthetic protocol was based on a previously published procedure. ${ }^{27}$ A suspension of trans-4-(aminomethyl)cyclohexane carboxylic acid $(786 \mathrm{mg}, 5.0 \mathrm{mmol})$ and maleic anhydride (490 mg, $5.0 \mathrm{mmol}$ ) in $25 \mathrm{~mL}$ of anhydrous dimethylformamide (DMF) in a 100 
$\mathrm{mL}$ round-bottom flask was stirred at room temperature for $6 \mathrm{~h}$. The resulting solution was cooled to $0{ }^{\circ} \mathrm{C}$, and sym-collidine $(1.4 \mathrm{~mL}, 10.5 \mathrm{mmol})$ was added dropwise. In a second round-bottom flask $(50 \mathrm{~mL}), N$-hydroxysuccinimide $(2.3 \mathrm{~g}, 20 \mathrm{mmol})$ was dissolved in anhydrous DMF $(25 \mathrm{~mL})$ and cooled to $0{ }^{\circ} \mathrm{C}$. To the $N$-hydroxysuccinimide solution stirred at $0{ }^{\circ} \mathrm{C}, 2.8 \mathrm{~mL}(20 \mathrm{mmol})$ of trifluoroacetic anhydride was added dropwise, and the mixture was allowed to stir for $10 \mathrm{~min}$. To this mixture, sym-collidine $(2.7 \mathrm{~mL}, 20 \mathrm{mmol})$ was added dropwise, and the mixture was allowed to stir for $10 \mathrm{~min}$. This mixture was then added dropwise to the first solution over a course of $1-2 \mathrm{~h}$ by cannula, maintaining both flasks at 0 ${ }^{\circ} \mathrm{C}$. The combined reaction mixture was allowed to return to room temperature, and the reaction was allowed to proceed overnight. The reaction mixture was diluted with $60 \mathrm{~mL}$ of DCM and washed with $1 \mathrm{M}$ aqueous hydrochloric acid $(3 \times 25 \mathrm{~mL})$. The organic layer was dried over magnesium sulfate, filtered, and concentrated by rotary evaporation. The residue was resuspended in minimal diethyl ether, and the white precipitate was collected by vacuum filtration. The precipitate was washed with diethyl ether $(2 \times 50 \mathrm{~mL})$ and dried under vacuum to yield succinimidyl-4-( $N$-maleimidomethyl)-cyclohexane-1-carboxylate (SMCC) as a white solid (1.0 g, $3.0 \mathrm{mmol}, 60 \%$ yield). ${ }^{1} \mathrm{H} \mathrm{NMR}\left(500 \mathrm{MHz}, \mathrm{CDCl}_{3}\right) \delta \mathrm{ppm}$ : $6.71(\mathrm{~s}, 2 \mathrm{H}), 3.39$ (d, $J=7.1 \mathrm{~Hz}, 2 \mathrm{H}), 2.82(\mathrm{~m}, 4 \mathrm{H}), 2.57$ (tt, $J=12.2,3.5 \mathrm{~Hz}, 1 \mathrm{H}), 2.16(\mathrm{~m}$, $2 \mathrm{H}), 1.79(\mathrm{~m}, 2 \mathrm{H}), 1.72$ (ddd, $J=11.3,7.6,3.8 \mathrm{~Hz}, 1 \mathrm{H}), 1.53(\mathrm{qd}, J=13.0,3.4 \mathrm{~Hz}, 2 \mathrm{H})$, 1.06 (qd, $J=12.8,3.4 \mathrm{~Hz}, 2 \mathrm{H}) ;{ }^{13} \mathrm{C}$ NMR $\left(126 \mathrm{MHz}, \mathrm{CDCl}_{3}\right) \delta \mathrm{ppm}: 170.94,170.55$, $169.13,133.98,43.34,40.34,36.02,29.25,27.95,25.54$.

\section{Synthesis of Diene- and Dienophile-Functionalized Multi-Arm PEGs.}

Multi-arm PEG macromers were functionalized with furan, methylfuran, and fulvene dienes and maleimide dienophiles by standard amide coupling chemistries using HATU- or NHSactivated carboxylic acid precursors, as described in detail in the Supporting Information. PEG-maleimide/tetrazine was prepared by reacting 8-arm PEG-amine with SMCC and methyltetrazine-NHS ester (Click Chemistry Tools), as described in the Supporting Information. The polymers were purified by precipitation in diethyl ether followed by either dialysis against Milli-Q-grade water or aqueous washes. Detailed synthetic protocols for each multi-arm PEG variant are included in the Supporting Information. Successful PEG functionalization was confirmed by ${ }^{1} \mathrm{H}$ NMR (Supporting Information).

\section{Preparation of Fulvene-Modified ELP.}

Engineered ELPs containing the integrin-binding RGD motif were produced by standard recombinant protein expression techniques and purified as previously described. ${ }^{28,29}$ The primary amines in the ELPs were sequentially modified with cysteic acid (to increase solubility) and 4-(cyclopenta-2,4-dien-1-ylidene)pentanoic acid (fulvene) using standard peptide synthesis approaches. Experimental details and ${ }^{1} \mathrm{H}$ NMR characterization of the functionalized proteins are provided in the Supporting Information.

\section{Rheological Characterization.}

Functionalized multi-arm PEGs were dissolved separately into phosphate-buffered saline (PBS) at a concentration of $50 \mathrm{mg} / \mathrm{mL}$. Hydrogels were formed by mixing maleimide-PEGs with furan-, methylfuran-, or fulvene-PEGs at a 1:1 volume ratio. Hydrogels were cast directly on the rheometer stage. Rheological characterization was performed using an ARG2 
rheometer fitted with a $20 \mathrm{~mm}, 1^{\circ}$ cone geometry. To prevent dehydration, a solvent trap was used for all experiments. For runs longer than a few hours, the samples were additionally surrounded by mineral oil to further prevent drying. Gelation time sweeps were performed at $1 \mathrm{~Hz}$ oscillatory frequency, $5 \%$ strain, and $37^{\circ} \mathrm{C}$. The gelation point was identified by finding the time at which $\tan (\delta)$ reached a local maximum (Figure S1). Following complete gelation, frequency sweeps from 0.1 to $10 \mathrm{~Hz}$ were performed at $10 \%$ strain and $37{ }^{\circ} \mathrm{C}$. For ELP-fulvene hydrogels, ELP-fulvene was dissolved to $50 \mathrm{mg} / \mathrm{mL}$ in PBS on ice and mixed with 4-arm maleimide-PEG $(50 \mathrm{mg} / \mathrm{mL})$ at a $1: 1$ volume ratio. Hydrogels were cast directly on the rheometer stage pre-cooled to $4{ }^{\circ} \mathrm{C}$. During the gelation time sweep, the stage was held at $4{ }^{\circ} \mathrm{C}$ for $10 \mathrm{~min}$ to prevent thermal aggregation of the ELP, and then the temperature was increased to $37{ }^{\circ} \mathrm{C}$ at a rate of $3{ }^{\circ} \mathrm{C} / \mathrm{min}$ and held at $37{ }^{\circ} \mathrm{C}$ for the remainder of the run. As above, gelation time sweeps were performed at $1 \mathrm{~Hz}$ oscillatory frequency and $5 \%$ strain.

\section{Hydrogel Degradation Study.}

PEG components were dissolved into PBS and mixed to form hydrogels as described above. Hydrogels $(50 \mu \mathrm{L})$ were cast into pre-weighed microcentrifuge tubes and cross-linked in a $37^{\circ} \mathrm{C}$ incubator. Fulvene gels were given $4 \mathrm{~h}$ to ensure complete cross-linking, while furan and methylfuran gels were cross-linked for $\sim 24 \mathrm{~h}$. PBS $(1 \mathrm{~mL})$ was added to the microcentrifuge tubes, and the samples were maintained at $37^{\circ} \mathrm{C}$. To assess hydrogel degradation, PBS was carefully aspirated and excess liquid wicked away with a Kimwipe prior to measuring the wet mass of hydrogels. After measuring the wet mass, the samples were frozen at $-80^{\circ} \mathrm{C}$ and lyophilized. The dry mass of the hydrogels was then measured. Mass loss over time was determined from the hydrogel dry mass measurements. Hydrogel mass swelling ratios were calculated by dividing the wet mass by the corresponding dry mass for each gel. For 4-arm PEG-fulvene gels, time points were collected at 0, 1, 3, 7, 14, 21, and 28 days and at 2, 3, and 4 months. For 4-arm PEG-furan gels, time points were collected at $0,1,2$, and 3 days, and for 4-arm PEG- methylfuran gels, time points were collected at $0,1,2,3$, and 4 days. For 8-arm PEG-fulvene gels, time points were collected at $1,7,14$, and 28 days and at 2, 3, and 4 months. For 8-arm PEG-furan gels, time points were collected at $1,7,14,28$, and 49 days.

The mechanism of hydrogel degradation was assessed by ${ }^{1} \mathrm{H}$ NMR analysis. Deuterated phosphate buffer $(\mathrm{pD} \approx 7.4 ; 100 \mathrm{mM} ; 10 \times)$ was prepared by dissolving sodium phosphate dibasic $\left(\mathrm{Na}_{2} \mathrm{HPO}_{4} ; 51.1 \mathrm{mg}\right)$ and sodium phosphate monobasic $\left(\mathrm{NaH}_{2} \mathrm{PO}_{4} ; 16.8 \mathrm{mg}\right)$ in deuterium oxide $(5 \mathrm{~mL})$. The phosphate buffer was diluted to $10 \mathrm{mM}$ in deuterium oxide and used to dissolve the diene- and dienophile-modified 4-arm PEGs to $50 \mathrm{mg} / \mathrm{mL}$. The furan, methylfuran, or fulvene PEG solution was mixed in a 1:1 volumetric ratio with the maleimide PEG solution and added to an NMR tube. The ${ }^{1} \mathrm{H}$ NMR spectra were immediately collected to obtain pre-reaction data. The tubes were subsequently maintained at $37^{\circ} \mathrm{C}$, and NMR spectra were collected at 1,7 , and 14 days to assess gelation and subsequent degradation.

\section{Cell Culture.}

Human bone marrow-derived mesenchymal stromal cells (hMSCs) were purchased from Lonza. The cells were expanded in high glucose Dulbecco's modified Eagle's medium 
supplemented with $10 \%$ fetal bovine serum, $1 \%$ GlutaMAX, and $1 \%$ penicillin/streptomycin (Gibco) on tissue culture-treated plastic in a $37{ }^{\circ} \mathrm{C}, 5 \% \mathrm{CO}_{2}$ incubator. Culture medium was changed every two days and the cells were passaged approximately weekly using trypsin/ EDTA (Gibco). The cells were used at passage 8.

\section{Cell Settling Assay.}

The protocol to assess cell settling in DA cross-linked hydrogels was based on a previously published procedure. ${ }^{30} \mathrm{hMSC}$ sere lifted by trypsinization, washed with PBS, and lightly fixed with $1 \%$ paraformaldehyde in PBS. The cells were stained with Hoechst DNA dye to visualize nuclei by fluorescence microscopy. The cells were pelleted by centrifugation, washed to remove unbound dye, and pelleted again. The cell pellet was resuspended in a 5\% $(\mathrm{w} / \mathrm{v})$ solution of 4-arm PEG-maleimide in PBS to a concentration of $4 \times 10^{6}$ cells $/ \mathrm{mL}$. The cell suspension was then mixed in a 1:1 volumetric ratio with a $5 \%(\mathrm{w} / \mathrm{v})$ solution of either 4-arm PEG-furan, 4-arm PEG-methylfuran, or 4-arm PEG-fulvene in PBS to afford a final cell concentration of $2 \times 10^{6}$ cells $/ \mathrm{mL}$. $70 \mu \mathrm{L}$ of the pregel solution was then added to a microcuvette ( $n=3$ per condition) and allowed to gel upright at $37{ }^{\circ} \mathrm{C}$ overnight. The following day, the cuvette was laid on its side and imaged on a Leica SPE confocal microscope. The degree of cell settling was quantified by dividing the images into 4 segments along the vertical axis, counting the number of cells per segment, and then calculating the sedimentation coefficient ( $\delta$ in eq 1 ) as previously described ${ }^{30}$

$$
\delta=n \frac{\sum c_{i}^{2}}{\left(\sum c_{i}\right)^{2}}
$$

where $n$ is the number of image segments and $c_{i}$ is the cell density per segment. Cell density was approximated by measuring the grayscale pixel intensity as a function of the $z$-axis position using ImageJ. The image was divided into 4 equal segments, and the cell density per segment was given by summing the pixel intensity values within that segment.

\section{Cell Encapsulation in DA ELP Hydrogels.}

After trypsinization, hMSCs were resuspended in a 5\% (w/v) solution of ELP-fulvene in PBS to a concentration of $4 \times 10^{6}$ cells $/ \mathrm{mL}$ and maintained on ice. The cell suspension was mixed in a 1:1 volumetric ratio with a 5\% (w/v) solution of 4-arm PEG-maleimide in PBS to afford a final cell concentration of $2 \times 10^{6}$ cells $/ \mathrm{mL}$. To enable facile handling of hydrogels in culture and during fluorescence microscopy analysis, gels were cast into custom cylindrical silicone molds $(8 \mathrm{~mm}$ diameter $\times 0.5 \mathrm{~mm}$ height $)$ prepared from $0.5 \mathrm{~mm}$ thick silicone insulator (Electron Microscopy Sciences) that was oxygen plasma bonded to no. 1 glass coverslips, as previously described. ${ }^{31}$ The pregel mixture was dispensed into the molds in 24-well tissue culture plates and maintained on ice for $10 \mathrm{~min}$ to prevent thermal aggregation of the ELP. The plates were then moved to room temperature for $5 \mathrm{~min}$ before being transferred to a $37{ }^{\circ} \mathrm{C}$ tissue culture incubator for $20 \mathrm{~min}$ to complete gelation. The wells were then filled with cell culture medium and maintained in a $37{ }^{\circ} \mathrm{C}, 5 \% \mathrm{CO}_{2}$ incubator. Culture medium was changed every two days. 


\section{Cell Viability Assay.}

The viability of hMSCs encapsulated in DA ELP hydrogels was assessed both $1 \mathrm{~h}$ and 7 days after encapsulation. At each time point, 4 gels were stained with Live/Dead reagents (Thermo Fisher Scientific) diluted into phenol red-free culture medium (1:2000 dilution for calcein-AM, 1:500 for ethidium homodimer). After incubating in the dye solution for $40 \mathrm{~min}$ at $37^{\circ} \mathrm{C}$, the gels were washed twice with phenol red-free culture medium and imaged on a Leica SPE confocal microscope. The images were processed using ImageJ software (NIH).

\section{Cell Morphology Assay.}

The morphology of hMSCs encapsulated in DA ELP hydrogels was assessed 7 days after encapsulation. Samples were fixed with 4\% paraformaldehyde in PBS, washed twice with PBS, and then permeabilized with $0.25 \%$ Triton X-100 in PBS (PBST). Samples were stained with FITC-phalloidin to label F-actin and Hoechst to label DNA and then washed three times with PBST. Samples were mounted onto coverslips by placing a drop ( $20 \mu \mathrm{L})$ of antifade mounting medium (Thermo Fisher Scientific) on the coverslip and inverting the silicone molds containing gels onto the drop of mounting medium. The mounted samples were imaged on a Leica SPE confocal microscope.

\section{Orthogonal Chemical Functionalization Assay.}

To demonstrate that fulvene-based DA cross-linking is amenable to the use of other "click" reactions to subsequently functionalize hydrogels, gels were prepared by mixing a $5 \%(\mathrm{w} / \mathrm{v})$ solution of ELP-fulvene in PBS in a 1:1 volumetric ratio with a 5\% (w/v) solution of 8-arm PEG-maleimide (negative control) or 8-arm PEG-maleimide/tetrazine in PBS. Gels (15 $\mu \mathrm{L}$ ) were cast in microcentrifuge tubes and maintained on ice for $10 \mathrm{~min}$ to prevent thermal aggregation of the ELP. The samples were then moved to room temperature for $5 \mathrm{~min}$ before being placed at $37^{\circ} \mathrm{C}$ for $1 \mathrm{~h}$ to complete gelation. The gels were allowed to equilibrate in PBS $(0.5 \mathrm{~mL})$ for $30 \mathrm{~min}$ at $37^{\circ} \mathrm{C}$. The gels were then incubated for $1 \mathrm{~h}$ in PBS with or without a bicyclononyne (BCN)-functionalized far-red dye (CF640R-BCN; Biotium; $10 \mu \mathrm{M}$ ) at $37^{\circ} \mathrm{C}$. The supernatant was discarded, and the gels were washed with $0.5 \mathrm{~mL}$ PBS $(3 \times 15$ $\mathrm{min}$ ). The gels were then degraded by incubation with $0.5 \%$ trypsin/EDTA (Gibco) at $37{ }^{\circ} \mathrm{C}$ and mechanical disruption by sonication. The fluorescence intensity of dye bound in the hydrogel network was measured with a Molecular Devices SpectraMax M2 plate reader.

\section{Statistical Analysis.}

For comparisons between two experimental groups, two-tailed Student's $t$-tests were used to determine statistical significance. For comparisons among more than two experimental groups, one-way analysis of variance (ANOVA) with Bonferroni post-hoc testing to account for multiple comparisons was used to determine statistical significance. For the gelation point experiment using 8-arm PEGs, a single sample, two-tailed Student's $t$-test was used to test the null hypothesis that the furan gel formed at the same rate as the fulvene gel (i.e., that the gelation point for the furan gels occurred within the $\sim 30 \mathrm{~s}$ it took to start the rheometer after mixing the precursor solutions). A $P$-value of less than 0.05 was considered to be statistically significant. 


\section{RESULTS AND DISCUSSION}

\section{Improving the Gelation Kinetics of DA Cross-linking for Cell Encapsulation.}

The first limitation with current DA reaction pairs used to cross-link hydrogels is their relatively slow gelation kinetics. To assess whether substituting commonly used furan moieties with fulvenes would enhance the gelation rate of hydrogels, separate 4-arm PEG components were modified with maleimide dienophiles and either furan, methylfuran, or fulvene dienes by standard amide coupling chemistry (Figure 2A). The diene and dienophile PEGs were mixed together in a 1:1 molar ratio with a final polymer content of $5 \%(\mathrm{w} / \mathrm{v})$ and subjected to oscillatory shear rheology to determine gelation kinetics (Figure 2B). Gelation kinetics were assessed by two metrics: time until the critical gelation point (i.e., the point at which a percolating network is first formed; Figure S1) and time until the hydrogels reached half their maximal storage modulus. Whereas the furan and methylfuran-based hydrogels took $\sim 10$ and $\sim 7 \mathrm{~h}$, respectively, to reach the critical gelation point, fulvene-based hydrogels reached the critical gelation point in less than $20 \mathrm{~min}$, a greater than 10 -fold improvement (Figure 2C). Similarly, half-maximal modulus was achieved in fulvene gels in $\sim 40 \mathrm{~min}$, compared to greater than $10 \mathrm{~h}$ for the furan and methylfuran gels (Figure 2D). Consistent with previous reports, the methylfuran-based gels cross-link more rapidly than unsubstituted furan-based gels (Figure 2B,C). ${ }^{22}$

Gelation kinetics are a function of multiple parameters in addition to the reaction rate of the cross-linking groups, such as the concentration of the polymeric components and the number of functional groups per component. Percolation theory predicts that for a given concentration of polymeric components, an increase in the number of reactive groups per polymer will achieve a percolating network more rapidly during cross-linking, thereby decreasing the time required to cross-link the network. Accordingly, we also measured the gelation kinetics of DA hydrogels based on 8-arm PEGs. To assess the two extremes of reaction rates, we compared 8 -arm PEGs functionalized with furan and fulvene dienes (Figure 2E). Consistent with our results with 4-arm PEGs, fulvene-based gels cross-linked much more rapidly than furan-based gels. The furan gels reached the critical gelation point in $\sim 2 \mathrm{~h}$, whereas the fulvene gels crossed the gelation point before the rheometer could start collecting data (less than $\sim 30 \mathrm{~s}$ ) (Figure $2 \mathrm{~F}$ ). Similarly, furan gels reached their halfmaximal storage modulus in $\sim 5.5 \mathrm{~h}$, while fulvene gels reached a half-maximal storage modulus in less than $10 \mathrm{~min}$ (Figure 2G). As expected, for both furan and fulvene gels, 8arm PEGs reached their percolation threshold much faster than the corresponding 4-arm PEGs due to the increased number of reactive groups per PEG macromer. Indeed, previous studies have shown that heavily functionalized polysaccharides can enable sufficiently rapid gelation even with furan and methylfuran dienes to be useful for cell encapsulation. ${ }^{15,16,22}$ The addition of fulvene-based DA cross-linking now expands the utility of this chemistry to other hydrogel network architectures, such as 4-arm PEGs, that are commonly used as 3D tissue engineering scaffolds and drug delivery platforms.

To demonstrate that fulvene-based DA hydrogels exhibit suffciently rapid gelation kinetics to enable homogeneous cell encapsulation, a cell sedimentation assay was performed using human mesenchymal stromal cells (hMSCs, also commonly referred to as mesenchymal 
stem cells) and 4-arm PEG macromers. The cells were lightly fixed, stained with Hoechst to label the nuclei, and suspended in a 1:1 volumetric mixture of maleimide PEG and diene PEG at $5 \%(\mathrm{w} / \mathrm{v})$ total polymer content. The mixture was transferred to a microcuvette and allowed to cross-link for $24 \mathrm{~h}$ at $37^{\circ} \mathrm{C}$. The gels were imaged by confocal microscopy, and cell sedimentation was quantified as a "sedimentation coefficient" as defined previously. ${ }^{30}$ This sedimentation coefficient is the second moment average divided by the first moment average, which is analogous to the dispersity index for polymeric materials. Thus, a coefficient of 1 indicates a perfectly homogeneous distribution of cells throughout the volume of the gel, whereas coefficients greater than 1 indicate that sedimentation has occurred. As expected from their slow gelation kinetics, furan and methylfuran gels resulted in significant cell sedimentation, with coefficients of $\sim 3$ (Figure 3). In contrast, sedimentation coefficients for fulvene gels were $\sim 1$, indicating a homogeneous cell distribution throughout the hydrogels (Figure 3 ). Thus, fulvene gels cross-link rapidly enough to maintain a homogeneous cell distribution, even in hydrogels prepared from polymers with low degrees of diene and dienophile functionalization.

\section{Improving the Stability of DA Cross-linked Hydrogels for Cell Culture.}

A second significant limitation in using DA-based hydrogels as 3D cell culture platforms is the relatively rapid degradation rate of maleimide-furan gels under physiological conditions. DA reactions are reversible, ${ }^{21,32-34}$ with retro-DA reactions liberating the original maleimide groups at cross-link points. While these maleimides can react again with the corresponding furans to re-form the cross-link, the maleimide groups are also susceptible to hydrolysis and can degrade to a maleamic acid that does not appreciably participate in network cross-links due to its significantly slower DA reaction kinetics. ${ }^{19}$ Thus, over time, the number of crosslinks is decreased, resulting in increased hydrogel swelling that eventually results in dissolution of the network. Previous approaches to decrease hydrolysis involved adding hydrophobic spacers to the maleimide to decrease water accessibility and increasing the number of reactive groups per macromer to maintain a percolating network for a longer duration. ${ }^{11}$ Nevertheless, such gels still exhibited significant increases in swelling, indicating a decrease in cross-link density over time that is directly linked to changes in other important hydrogel properties including stiffness and nutrient transport that can alter cell phenotype.

To assess the stability of fulvene-based DA hydrogels, the mass swelling ratio and dry mass of hydrogels were monitored over time (Figure 4). Hydrogels were prepared by mixing multi-arm PEGs functionalized with maleimide dienophiles and furan, methylfuran, or fulvene dienes as described above. The gels were maintained in PBS at $37{ }^{\circ} \mathrm{C}$, and samples were collected at defined time points. Consistent with previous results, ${ }^{19}$ gels formed from 4-arm PEG modified with furan and methylfuran dienes exhibited substantial changes in swelling behavior and were completely degraded by 3 and 4 days after cross-linking, respectively (Figure 4B,C). Strikingly, fulvene-based DA hydrogels exhibited markedly improved stability, with only modestly increased swelling and negligible mass loss after 4 months under physiological conditions (Figure 4B,C). Accordingly, rheological characterization of the three hydrogels revealed significant time-dependence in the mechanical properties of freshly prepared furan and methylfuran hydrogels, but not in 
fulvene hydrogels, suggesting that cross-links formed by furan-based moieties are more dynamic and thus expected to be less stable over time (Figure S2).

The mechanism of hydrogel degradation via a retro-DA reaction and subsequent hydrolysis of the maleimide dienophile was confirmed by NMR (Figures S3 and S4). Consistent with the gelation kinetics data obtained from rheological analysis (Figure 2), NMR reveals that the DA cross-linking reaction approaches completion within $24 \mathrm{~h}$ for all hydrogel formulations, as indicated by the disappearance of the maleimide resonance peak. After further maintaining the gels in phosphate buffer at $37^{\circ} \mathrm{C}$, the peaks corresponding to the DA adduct decreased from 1 to 7 to 14 days in the furan- and methylfuran-based gels (Figure S3), while the adduct signal was stable in the fulvene gels (Figure S4), indicating reversibility of the furan and methylfuran DA cross-links. Further, NMR peaks corresponding to the hydrolyzed maleimide appeared and increased in intensity throughout this time interval for the furan and methylfuran gels (Figure S3), indicating that the maleimides existing in equilibrium with the DA adduct were hydrolyzing and thereby decreasing the number of available cross-link sites over time.

Consistent with this degradation mechanism, increasing the degree of macromer functionalization by using 8-arm PEG had the expected effect of increasing stability by increasing network connectivity (Figure 4D-F). 8-arm PEG hydrogels formed from furan macromers exhibited more gradual swelling compared to 4-arm gels and were fully degraded by 7 weeks (Figure 4E,F). 8-arm fulvene gels continued to demonstrate excellent stability, with negligible changes in both swelling and dry mass after 4 months (Figure $4 \mathrm{E}, \mathrm{F})$. Thus, fulvene-based DA gels are attractive as 3D cell culture platforms to culture cells for long durations with consistent microenvironmental parameters.

These results demonstrate that the choice of DA reaction pair and the degree of functionality of the polymeric components dictate several crucial hydrogel properties that determine the suitability of the gel system for a given application. For instance, rapidly cross-linking fulvene gels best maintain a homogenous cell distribution, making them useful as 3D cell culture materials. The 8-arm fulvene gels cross-link on a very rapid time scale (seconds to minutes), suggesting that highly functionalized fulvene-polymers may be useful in a dualcomponent bio-ink for 3D printing of cell-laden constructs that require fast gelation kinetics to retain their printed shape. The chemical stability of the cross-links in the fulvene gels makes these materials suitable for cell culture applications in which the stiffness must be maintained within a specific range to expand or differentiate mechanosensitive stem cells. 35,36 In contrast, other cell types that require gradual degradation of the hydrogel material in order to grow, such as intestinal stem cells, ${ }^{37}$ may benefit from the addition of furan-based cross-links that are more susceptible to degradation. Furan-based networks may additionally be useful to control the release of cells or drugs for therapeutic applications by programming the rate of degradation into the network through selecting the identity and number of DA reaction pairs per polymeric component.

\section{Hybrid Protein-PEG DA Hydrogels for Cell Culture.}

Hydrogels used in 3D cell culture should present bioactive signals, such as cell-adhesive ligands and peptides that enable proteolytic degradation, to maintain high cell viability and 
to allow cell spreading and migration. ${ }^{38,39}$ To incorporate this additional functionality in DA cross-linked hydrogels, engineered ELPs were functionalized with fulvenes to enable crosslinking with 4-arm PEG-maleimide (Figure 5A). The ELPs used contain repeating celladhesive and structural elastin-like domains. ${ }^{40}$ The cell-adhesive domains contain an extended RGD peptide sequence derived from human fibronectin to enable integrinmediated cell adhesion, and the structural domain consists of the elastin-like VPGXG peptide sequence, wherein 1 out of 5 ' $\mathrm{X}$ ' residues are lysines to provide primary amines for functionalization with the fulvenes for cross-linking. Prior to the addition of the fulvene moieties, the lysine residues were sulfated to increase the solubility of the ELPs. The ELPs are additionally susceptible to proteolytic degradation, enabling cell-mediated remodeling of the hydrogel matrix. While the elastin-like sequence is largely insensitive to proteases other than dedicated elastases, ${ }^{41}$ we have previously shown that the extended cell-adhesive sequence in these materials can be cleaved by zinc metalloproteases. ${ }^{42}$ The resulting gels cross-link rapidly, reaching their critical gelation point in less than 25 min (Figure 5B). Thus, the rapid cross-linking of the fulvene-maleimide DA reaction also applies to hybrid protein-synthetic polymer hydrogels.

To assess the suitability of fulvene DA cross-linked gels as 3D cell culture platforms, hMSCs were encapsulated within hydrogels prepared from fulvene-functionalized ELPs and 4-arm PEG-maleimide. Cell viability was assessed both acutely at $1 \mathrm{~h}$ after encapsulation and longer-term at 7 days after encapsulation by a live/dead cell membrane integrity assay (Figure 5C,D). The cells remained highly viable (>96\%) following encapsulation, indicating that the DA cross-linking mechanism is well tolerated by cells. After 7 days in culture, the cells remained $\sim 90 \%$ viable, consistent with previous results culturing hMSCs in hydrogels with similar concentrations of RGD peptide. ${ }^{28,38}$ Furthermore, after 7 days, the hMSC cultures showed evidence of cell-mediated remodeling. Cell morphology was assessed by fixing the cell-laden hydrogels and staining the actin cytoskeleton with fluorescent phalloidin. Protrusions were observed extending from the cell bodies into the surrounding hydrogel material, indicative of cell adhesion and local degradation of the gel network (Figure 5E). This behavior is consistent with hMSCs cultured in other hydrogel systems with cell-remodelable adhesive ligands. ${ }^{43-45}$

\section{Orthogonal Functionalization of DA Cross-linked Hydrogels.}

Finally, to demonstrate that the DA cross-linking reaction can be used in combination with an orthogonal "click" reaction to selectively functionalize hydrogels, a tetrazine ligation (an inverse electron demand DA reaction) was used to covalently attach a fluorescent dye to the polymer network. Hydrogels were prepared from fulvene ELP and an 8-arm PEG that was modified with both maleimides and tetrazines (Figure 6A). The tetrazines should not react to an appreciable degree during the DA cross-linking reaction, as both fulvenes and tetrazines are dienes and, due to the inverse electron demand nature of the tetrazine ligation, the reaction between tetrazines and maleimides is very slow. ${ }^{46}$ Following cross-linking, the remaining tetrazines are available to react with a fluorescent dye bearing a $\mathrm{BCN}$ group. As a control, gels were also prepared from 8-arm PEG modified only with maleimides. The excess maleimides remaining after cross-linking should not appreciably react with the $\mathrm{BCN}$ bearing dye. Significant dye incorporation was observed only in hydrogels containing 
tetrazine moieties, as indicated by a $\sim 25$-fold increase in fluorescence intensity over background levels (Figure 6B). Importantly, minimal residual dye remained in the hydrogels with excess maleimides, demonstrating the selectivity of the two-step, orthogonal click reaction approach.

\section{CONCLUSIONS}

These results indicate that fulvene-maleimide DA reaction pairs are a suitable chemistry for cross-linking cell-encapsulating hydrogels. Fulvene-based cross-linking overcomes the two major limitations of previous DA gels by markedly increasing the gelation rate of the materials to prevent cell sedimentation and by significantly increasing the hydrolytic stability of the gels to maintain consistent material properties over time. Adding rapid DA cross-linking to the biomaterials science toolkit will expand the range of functionalities that can be incorporated into hydrogel mimics of the ECM. The orthogonality of the DA reaction to other commonly used click chemistries enables the development of well-controlled, multicomponent hydrogel networks for use as tissue engineering scaffolds, cell transplantation vehicles, and drug delivery systems.

\section{Supplementary Material}

Refer to Web version on PubMed Central for supplementary material.

\section{ACKNOWLEDGMENTS}

C.M.M. was supported by a Ruth L. Kirschstein NRSA F31 fellowship (F31 EB020502) from the National Institutes of Health (NIH), by the Siebel Scholars Program, and by the Stanford ChEM-H Postdoctoral Training Program in Quantitative Mechanobiology. This work was supported by funding from the NIH (R21 HL138042, U19 AI116484, R01 HL142718), NSF (DMR 1808415), and the Stanford Wu Tsai Neurosciences Institute. Part of this work was performed at the Stanford Nano Shared Facilities (SNSF), supported by the National Science Foundation under award ECCS-1542152.

\section{REFERENCES}

(1). Lee KY; Mooney DJ Hydrogels for Tissue Engineering. Chem. Rev 2001, 101, 1869-1880. [PubMed: 11710233]

(2). Ratner BD; Bryant SJ Biomaterials: Where We Have Been and Where We Are Going. Annu. Rev. Biomed. Eng 2004, 6, 41-75. [PubMed: 15255762]

(3). Madl CM; Heilshorn SC Engineering Hydrogel Microenvironments to Recapitulate the Stem Cell Niche. Annu. Rev. Biomed. Eng 2018, 20, 21-47. [PubMed: 29220201]

(4). Jiang Y; Chen J; Deng C; Suuronen EJ; Zhong Z Click Hydrogels, Microgels and Nanogels: Emerging Platforms for Drug Delivery and Tissue Engineering. Biomaterials 2014, 35, 49694985. [PubMed: 24674460]

(5). Azagarsamy MA; Anseth KS Bioorthogonal Click Chemistry: An Indispensable Tool to Create Multifaceted Cell Culture Scaffolds. ACS Macro Lett. 2013, 2, 5-9. [PubMed: 23336091]

(6). Medina SH; Schneider JP Chemical Ligations in the Design of Hydrogel Materials In Chemoselective and Bioorthogonal Ligation Reactions: Concepts and Applications; Algar WR, Dawson PE, Medintz IL, Eds.; Wiley-VCH Verlag GmbH \& Co. KGaA: Weinheim, Germany, 2017.

(7). Führmann T; Obermeyer J; Tator CH; Shoichet MS Click-Crosslinked Injectable Hyaluronic Acid Hydrogel Is Safe and Biocompatible in the Intrathecal Space for Ultimate Use in Regenerative Strategies of the Injured Spinal Cord. Methods 2015, 84, 60-69. [PubMed: 25846399] 
(8). Koehler KC; Anseth KS; Bowman CN Diels-Alder Mediated Controlled Release from a Poly(ethylene glycol) Based Hydrogel. Biomacromolecules 2013, 14, 538-547. [PubMed: 23311608]

(9). Koehler KC; Alge DL; Anseth KS; Bowman CN A Diels-Alder modulated approach to control and sustain the release of dexamethasone and induce osteogenic differentiation of human mesenchymal stem cells. Biomaterials 2013, 34, 4150-4158. [PubMed: 23465826]

(10). Fan M; Ma Y; Zhang Z; Mao J; Tan H; Hu X Biodegradable hyaluronic acid hydrogels to control release of dexamethasone through aqueous Diels-Alder chemistry for adipose tissue engineering. Mater. Sci. Eng., C 2015, 56, 311-317.

(11). Kirchhof S; Gregoritza M; Messmann V; Hammer N; Goepferich AM; Brandl FP Diels-Alder hydrogels with enhanced stability: First step toward controlled release of bevacizumab. Eur. J. Pharm. Biopharm 2015, 96, 217-225. [PubMed: 26253504]

(12). Bai X; Lü S; Cao Z; Ni B; Wang X; Ning P; Ma D; Wei H; Liu M Dual Crosslinked Chondroitin Sulfate Injectable Hydrogel Formed Via Continuous Diels-Alder (DA) Click Chemistry for Bone Repair. Carbohydr. Polym 2017, 166, 123-130. [PubMed: 28385214]

(13). Tan H; Rubin JP; Marra KG Direct Synthesis of Biodegradable Polysaccharide Derivative Hydrogels through Aqueous Diels-Alder Chemistry. Macromol. Rapid Commun 2011, 32, 905911. [PubMed: 21520481]

(14). Montiel-Herrera M; Gandini A; Goycoolea FM; Jacobsen NE; Lizardi-Mendoza J; Recillas-Mota M; Argüelles-Monal WM N-(furfural) chitosan hydrogels based on Diels-Alder cycloadditions and application as microspheres for controlled drug release. Carbohydr. Polym 2015, 128, 220 227. [PubMed: 26005158]

(15). Nimmo CM; Owen SC; Shoichet MS Diels-Alder Click Cross-Linked Hyaluronic Acid Hydrogels for Tissue Engineering. Biomacromolecules 2011, 12, 824-830. [PubMed: 21314111]

(16). Yu F; Cao X; Li Y; Zeng L; Zhu J; Wang G; Chen X Diels-Alder crosslinked HA/PEG hydrogels with high elasticity and fatigue resistance for cell encapsulation and articular cartilage tissue repair. Polym. Chem 2014, 5, 5116-5123.

(17). Rideout DC; Breslow R Hydrophobic Acceleration of Diels-Alder Reactions. J. Am. Chem. Soc 1980, 102, 7816-7817.

(18). Stewart SA; Backholm M; Burke NAD; Stöver HDH Cross-Linked Hydrogels Formed through Diels-Alder Coupling of Furan- and Maleimide-Modified Poly(methyl vinyl ether-alt-maleic acid). Langmuir 2016, 32, 1863-1870. [PubMed: 26800849]

(19). Kirchhof S; Brandl FP; Hammer N; Goepferich AM Investigation of the Diels-Alder reaction as a cross-linking mechanism for degradable poly(ethylene glycol) based hydrogels. J. Mater. Chem. B 2013, 1, 4855-4864. [PubMed: 32261167]

(20). Boutelle RC; Northrop BH Substituent Effects on the Reversibility of Furan-Maleimide Cycloadditions. J. Org. Chem 2011, 76, 7994-8002. [PubMed: 21866976]

(21). Froidevaux V; Borne M; Laborbe E; Auvergne R; Gandini A; Boutevin B Study of the DielsAlder and retro-Diels-Alder reaction between furan derivatives and maleimide for the creation of new materials. RSC Adv. 2015, 5, 37742-37754.

(22). Smith LJ; Taimoory SM; Tam RY; Baker AEG; Binth Mohammad N; Trant JF; Shoichet MS Diels-Alder Click-Cross-Linked Hydrogels with Increased Reactivity Enable 3D Cell Encapsulation. Biomacromolecules 2018, 19, 926-935. [PubMed: 29443512]

(23). St. Amant AH; Lemen D; Florinas S; Mao S; Fazenbaker C; Zhong H; Wu H; Gao C; Christie RJ; de Alaniz JR Tuning the Diels-Alder Reaction for Bioconjugation to Maleimide DrugLinkers. Bioconjugate Chem. 2018, 29, 2406-2414.

(24). Reutenauer P; Buhler E; Boul PJ; Candau SJ; Lehn J-M Room Temperature Dynamic Polymers Based on Diels-Alder Chemistry. Chem.-Eur. J 2009, 15, 1893-1900. [PubMed: 19132706]

(25). Wei Z; Yang JH; Du XJ; Xu F; Zrinyi M; Osada Y; Li F; Chen YM Dextran-Based Self-Healing Hydrogels Formed by Reversible Diels-Alder Reaction under Physiological Conditions. Macromol. Rapid Commun 2013, 34, 1464-1470. [PubMed: 23929621]

(26). Coşkun N; Erden I An Efficient Catalytic Method for Fulvene Synthesis. Tetrahedron 2011, 67, 8607-8614. [PubMed: 22021940] 
(27). Leonard NM; Brunckova J One-Pot Synthesis of Succinimidyl-4-(NMaleimidomethyl)Cyclohexane-1-Carboxylate (SMCC). Org. Prep. Proced. Int 2012, 44, 180 183.

(28). Madl CM; Katz LM; Heilshorn SC Bio-Orthogonally Crosslinked, Engineered Protein Hydrogels with Tunable Mechanics and Biochemistry for Cell Encapsulation. Adv. Funct. Mater 2016, 26, 3612-3620. [PubMed: 27642274]

(29). LeSavage BL; Suhar NA; Madl CM; Heilshorn SC Production of Elastin-Like Protein Hydrogels for Encapsulation and Immunostaining of Cells in 3D. J. Visualized Exp 2018, 135, No. e57739.

(30). Dubbin K; Tabet A; Heilshorn SC Quantitative Criteria to Benchmark New and Existing Bio-Inks for Cell Compatibility. Biofabrication 2017, 9, 044102. [PubMed: 28812982]

(31). Lampe KJ; Antaris AL; Heilshorn SC Design of Three-Dimensional Engineered Protein Hydrogels for Tailored Control of Neurite Growth. Acta Biomater. 2013, 9, 5590-5599. [PubMed: 23128159]

(32). Gheneim R; Perez-Berumen C; Gandini A Diels-Alder Reactions with Novel Polymeric Dienes and Dienophiles: Synthesis of Reversibly Cross-Linked Elastomers. Macromolecules 2002, 35, 7246-7253.

(33). Cuvellier A; Verhelle R; Brancart J; Vanderborght B; Van Assche G; Rahier H The influence of stereochemistry on the reactivity of the Diels-Alder cycloaddition and the implications for reversible network polymerization. Polym. Chem 2019, 10, 473-485.

(34). Sanyal A Diels-Alder Cycloaddition-Cycloreversion: A Powerful Combo in Materials Design. Macromol. Chem. Phys 2010, 211, 1417-1425.

(35). Gilbert PM; Havenstrite KL; Magnusson KEG; Sacco A; Leonardi NA; Kraft P; Nguyen NK; Thrun S; Lutolf MP; Blau HM Substrate Elasticity Regulates Skeletal Muscle Stem Cell SelfRenewal in Culture. Science 2010, 329, 1078-1081. [PubMed: 20647425]

(36). Engler AJ; Sen S; Sweeney HL; Discher DE Matrix Elasticity Directs Stem Cell Lineage Specification. Cell 2006, 126, 677-689. [PubMed: 16923388]

(37). Gjorevski N; Sachs N; Manfrin A; Giger S; Bragina ME; Ordóñez-Morán P; Clevers H; Lutolf MP Designer Matrices for Intestinal Stem Cell and Organoid Culture. Nature 2016, 539, 560564. [PubMed: 27851739]

(38). Jongpaiboonkit L; King WJ; Murphy WL Screening for 3D Environments That Support Human Mesenchymal Stem Cell Viability Using Hydrogel Arrays. Tissue Eng., Part A 2009, 15, 343353. [PubMed: 18759676]

(39). Baker BM; Chen CS Deconstructing the third dimension-how 3D culture microenvironments alter cellular cues. J. Cell Sci 2012, 125, 3015-3024. [PubMed: 22797912]

(40). Straley KS; Heilshorn SC Independent Tuning of Multiple Biomaterial Properties Using Protein Engineering. Soft Matter 2009, 5, 114-124.

(41). Mithieux SM; Weiss AS Elastin. Adv. Protein Chem 2005, 70, 437-461. [PubMed: 15837523]

(42). Madl CM; LeSavage BL; Dewi RE; Dinh CB; Stowers RS; Khariton M; Lampe KJ; Nguyen D; Chaudhuri O; Enejder A; Heilshorn SC Maintenance of Neural Progenitor Cell Stemness in 3D Hydrogels Requires Matrix Remodelling. Nat. Mater 2017, 16, 1233-1242. [PubMed: 29115291]

(43). Huebsch N; Arany PR; Mao AS; Shvartsman D; Ali OA; Bencherif SA; Rivera-Feliciano J; Mooney DJ Harnessing Traction-Mediated Manipulation of the Cell/Matrix Interface to Control Stem-Cell Fate. Nat. Mater 2010, 9, 518-526. [PubMed: 20418863]

(44). Chaudhuri O; Gu L; Klumpers D; Darnell M; Bencherif SA; Weaver J; Huebsch N; Lippens E; Duda GN; Mooney DJ; Mooney DJ Hydrogels with Tunable Stress Relaxation Regulate Stem Cell Fate and Activity. Nat. Mater 2016, 15, 326-334. [PubMed: 26618884]

(45). Tong X; Yang F Sliding Hydrogels with Mobile Molecular Ligands and Crosslinks as 3D Stem Cell Niche. Adv. Mater 2016, 28, 7257-7263. [PubMed: 27305637]

(46). Pagel M; Meier R; Braun K; Wiessler M; Beck-Sickinger AG On-resin Diels-Alder reaction with inverse electron demand: an efficient ligation method for complex peptides with a varying spacer to optimize cell adhesion. Org. Biomol. Chem 2016, 14, 4809-4816. [PubMed: 27117044] 


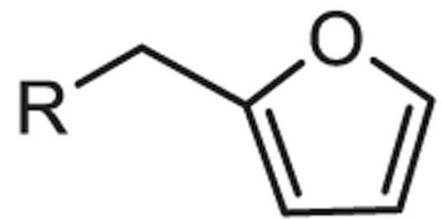

Furan

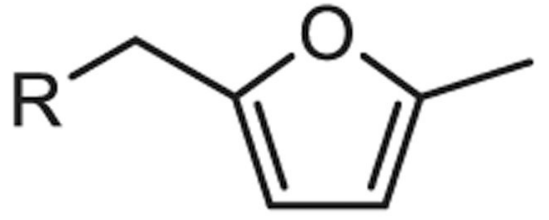

Me-Furan

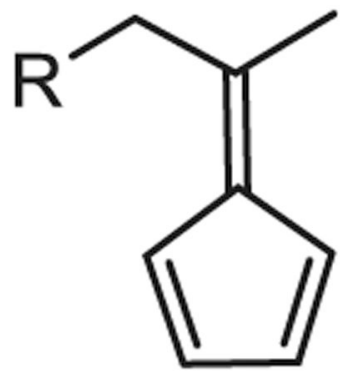

Fulvene

Figure 1.

Dienes used to prepare DA cross-linked hydrogels. 
A

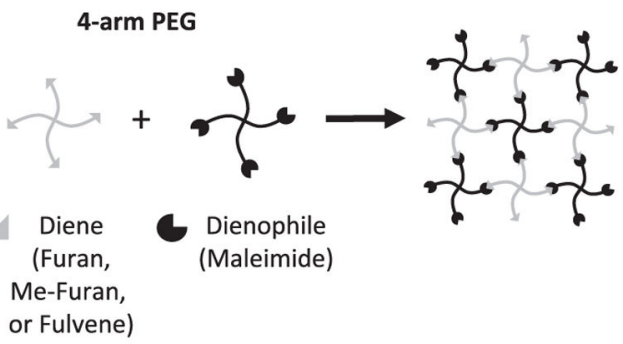

B

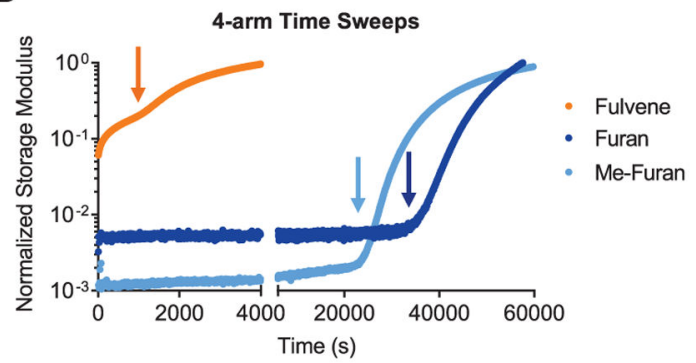

C

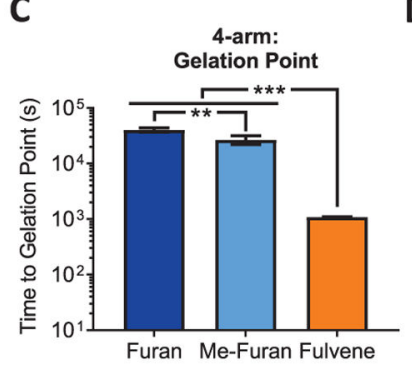

D

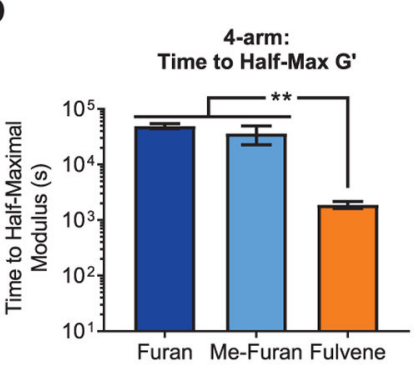

$\mathbf{E}$

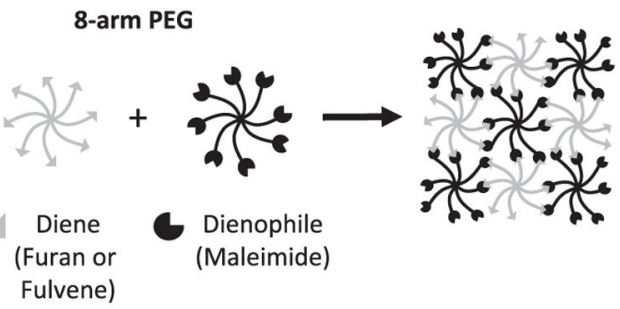

$\mathbf{F}$

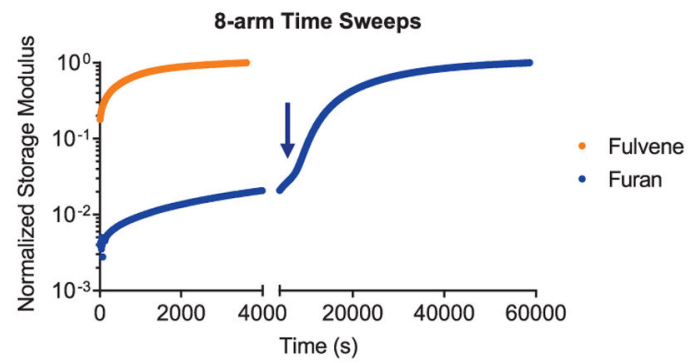

G

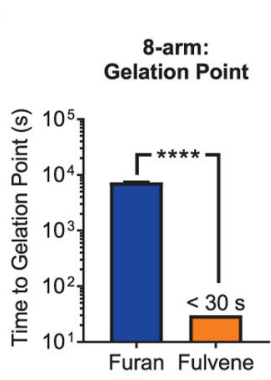

Figure 2.

Rapid cross-linking kinetics of fulvene-maleimide DA hydrogels. (A) 4-arm PEG hydrogels are prepared by mixing diene (furan, methylfuran, or fulvene) PEG with maleimide PEG.

(B) Gelation time sweeps of 4-arm PEG-based DA gels. Arrows indicate the critical gelation point. (C) Time to critical gelation point and (D) time to half-maximal storage modulus for 4-arm PEG-based DA gels. (E) 8-arm PEG hydrogels are prepared by mixing diene (furan or fulvene) PEG with maleimide PEG. (F) Gelation time sweeps of 8-arm PEG-based DA gels. The arrow indicates the critical gelation point for the furan gel. (G) Time to critical gelation point and $(\mathrm{H})$ time to half-maximal storage modulus for 8-arm PEG-based DA gels. In $\mathrm{C}$ and D, $* * P<0.01, * * * P<0.001$, one-way ANOVA with Bonferroni post-hoc test. In $\mathrm{G}$, $* * * * P$ $<0.0001$, one-sample Student's $t$-test with the null hypothesis that the gelation time for the furan gel is equivalent to the approximate gelation time for the fulvene gel $(\sim 30 \mathrm{~s})$. In $\mathrm{H}$, $* * * * P<0.0001$, two-tailed Student's $t$-test. Data are mean \pm s.d., $n=3-4$. 

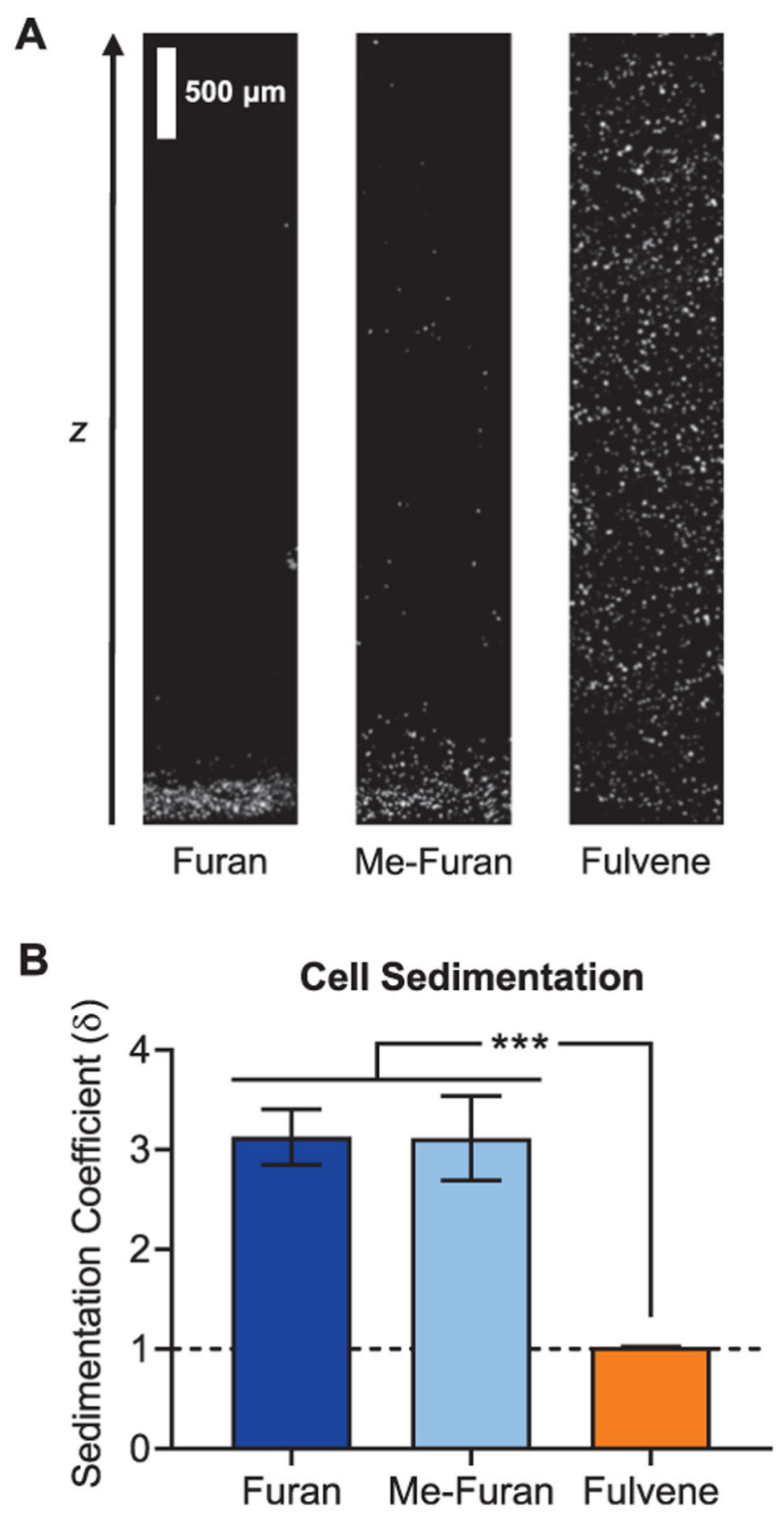

Figure 3.

Rapid cross-linking of fulvene-based DA gels prevents cell sedimentation. (A) Confocal fluorescence micrographs along the $z$-axis of 4-arm PEG-based DA hydrogels with encapsulated hMSCs. Prior to encapsulation, the hMSCs were lightly fixed with paraformaldehyde and stained with Hoechst to label the nuclei (white). (B) Cell sedimentation quantified as the sedimentation coefficient for hMSCs encapsulated in 4-arm PEG-based DA gels cross-linked with furan, methylfuran, or fulvene dienes. Dashed line represents a perfectly homogeneous cell distribution. *** $P<0.001$, one-way ANOVA with Bonferroni post-hoc test. Data are mean \pm s.d., $n=3$. 
A

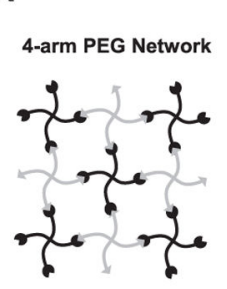

D

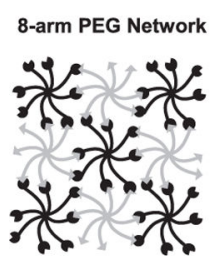

B

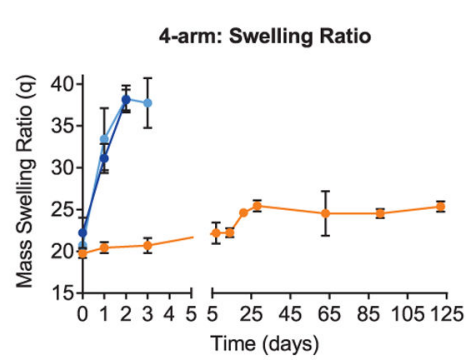

E

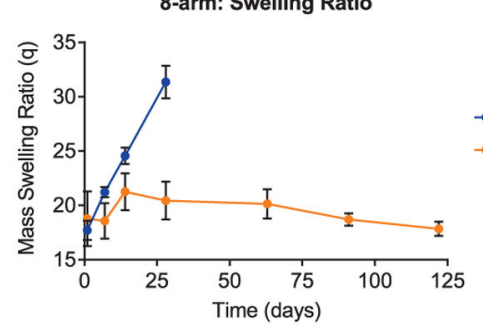

C

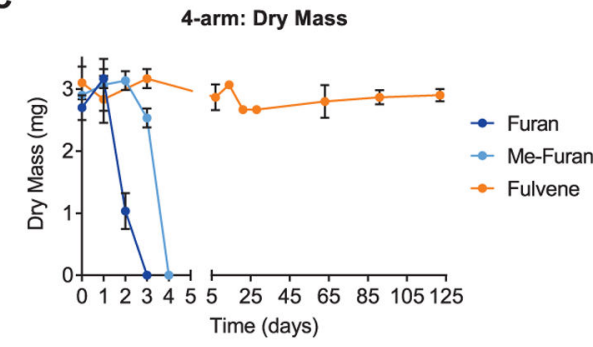

$\mathbf{F}$

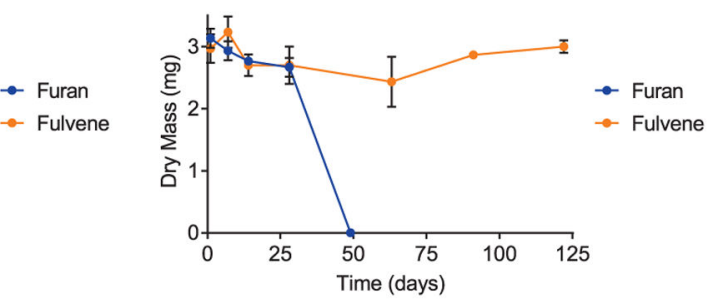

Figure 4.

Fulvene gels remain stable long-term under physiologically relevant conditions. (A)

Schematic of 4-arm PEG network. (B) Mass swelling ratio and (C) dry mass of 4-arm PEGbased DA hydrogel networks over time for samples maintained in PBS (pH 7.4) at $37{ }^{\circ} \mathrm{C}$. (D) Schematic of 8-arm PEG network. (E) Mass swelling ratio and (F) dry mass of 8-arm PEG-based DA hydrogel networks over time for samples maintained in PBS (pH 7.4) at 37 ${ }^{\circ} \mathrm{C}$. Data are mean \pm s.d., $n=3$ gels per time point. 
A

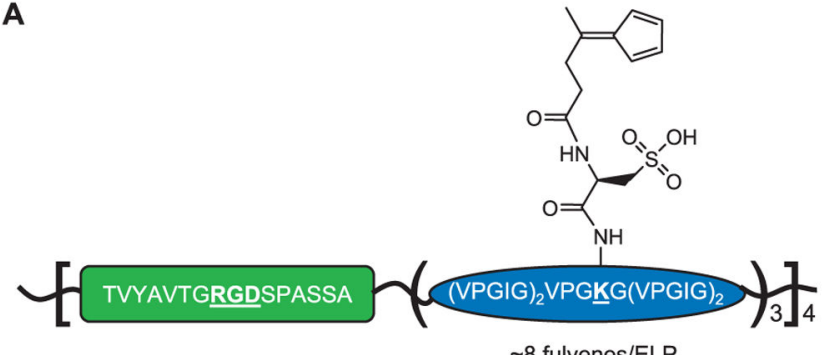

B

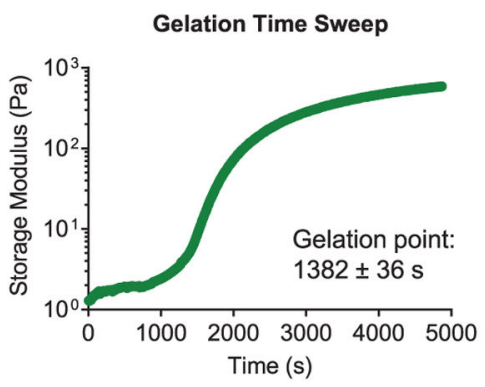

C Acute Viability $(1 \mathrm{~h})$

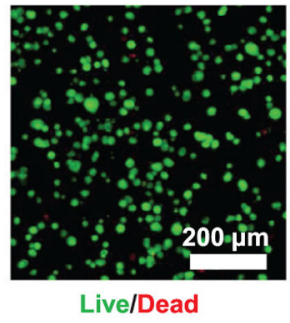

$96.4 \pm 0.4 \%$ Viable
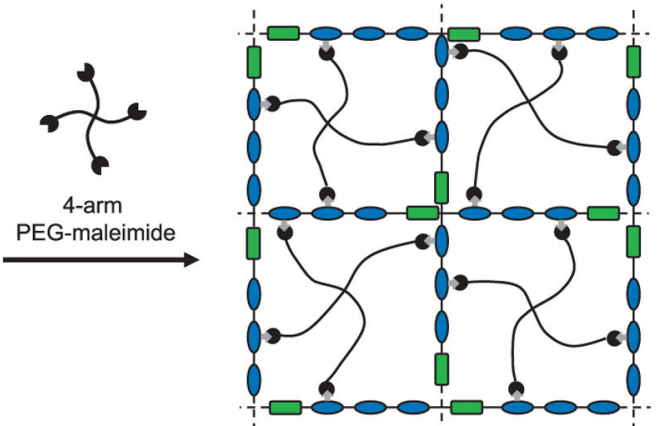

D Later Viability (7 d) E

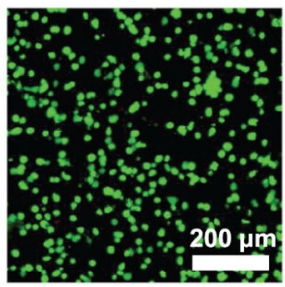

Live/Dead

$89.3 \pm 2.9 \%$ Viable
E Morphology (7 d)

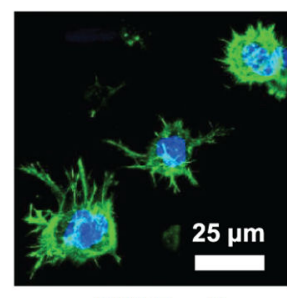

DNA/F-actin

Figure 5.

Engineered protein-PEG hybrid DA hydrogels support the culture of hMSCs. (A) Schematic of fulvene-modified ELPs containing a cell-adhesive RGD domain (green) and a structural elastin-like domain (blue). Upon mixing with a 4-arm PEG-maleimide crosslinker, the fulvene ELPs form hydrogel networks. (B) Gelation time sweep for ELP-PEG fulvene DA gels. Gelation point data are mean \pm s.d., $n=3$. Viability of hMSCs encapsulated in ELPPEG fulvene DA gels after (C) $1 \mathrm{~h}$ and (D) 7 days, measured by a live/dead cytotoxicity assay. Viability data are mean \pm s.d., $n=4$. (E) Confocal fluorescence micrograph showing spreading of hMSCs cultured in ELP-PEG fulvene DA gels for 7 days. The actin cytoskeleton was stained with phalloidin (green) and the nuclei were stained with Hoechst (blue). 
A

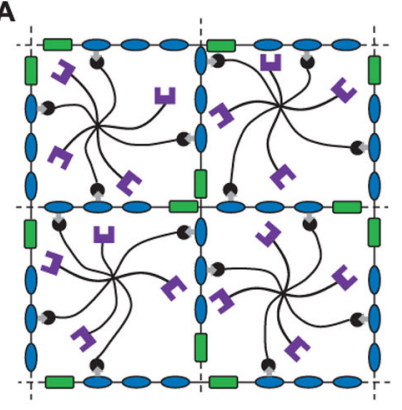

(CF640R-BCN)

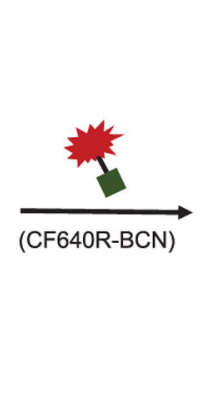

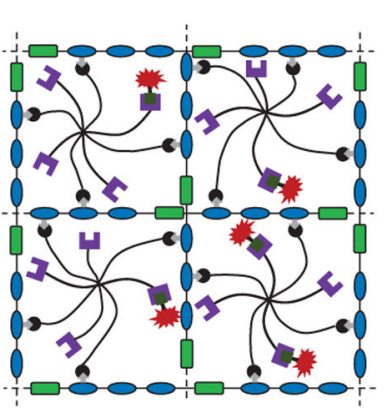

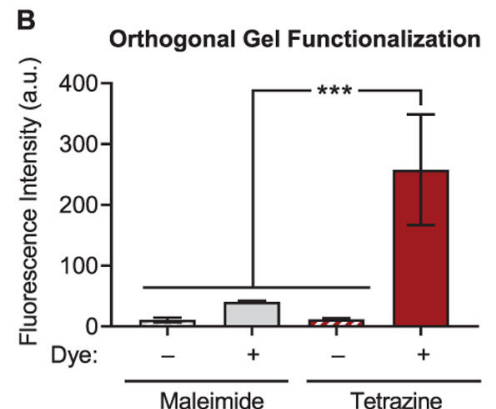

Figure 6.

Orthogonal functionalization of DA cross-linked hydrogels using tetrazine ligation. (A) Schematic depicting covalent modification of DA hydrogels prepared from ELP-fulvene and 8-arm PEG functionalized with maleimides (black) and tetrazines (purple) with a tetrazinereactive dye (CF640R-BCN, red). (B) Quantification of fluorescence intensity in gels with either excess maleimide or tetrazine moieties and either treated or untreated with tetrazinereactive dye. $* * * P<0.001$, one-way ANOVA with Bonferroni post-hoc test. Data are mean \pm s.d., $n=4$. 
<smiles>[R1]CC(C)=C1C=CC=C1</smiles><smiles>[R2]CN1C(=O)C=CC1=O</smiles><smiles>C=CC</smiles>

Fulvene (Diene)
Maleimide (Dienophile)

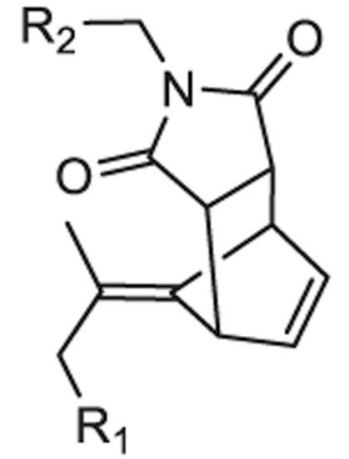

Diels-Alder Adduct

Scheme 1.

Fulvene-Maleimide DA Reaction 